\title{
Prediction of new-onset asthma and nasal allergy by skin prick test and RAST in a cohort of adults
}

\author{
Kerstin Gallmeier ${ }^{1}$, Eva Becker ${ }^{1}$, Anne Kirsten², Gabriele Wölke', \\ Olaf Manuwald ${ }^{3}$, Heike Meyer ${ }^{2}$, Helgo Magnussen², Dennis Nowak ${ }^{4}$ and \\ Joachim Heinrich ${ }^{1}$
}

\author{
Affiliations: \\ ${ }^{1}$ Helmholtz Zentrum München, Center for Environmental Health, Institute of Epidemiology I, Neuherberg, \\ ${ }^{2}$ Pulmonary Research Institute at Hospital Grosshansdorf, Center for Pneumology and Thoracic Surgery, \\ Grosshansdorf, \\ ${ }^{3}$ Institute for Environmental Medicine, Erfurt, and \\ 4Institute and Outpatient Clinic for Occupational, Social and Environmental Medicine, Ludwig-Maximilians- \\ University Munich, Munich, Germany.
}

Correspondence: J. Heinrich, Helmholtz Zentrum München, Center for Environmental Health, Institute of Epidemiology I, Ingolstädter Landstrasse 1, 85764 Neuherberg, Germany.

E-mail: heinrichahelmholtz-muenchen.de

ABSTRACT Limited information exists regarding the incidence and predictors of asthma and nasal allergy in adulthood. We determined the incidence rate of asthma and nasal allergy in adults and assessed the predictive value of skin prick tests (SPTs) and radioallergosorbent tests (RASTs) for these two outcomes.

Two German centres involved in the European Community Respiratory Health Survey conducted a follow-up assessment in 2012 of the baseline participants (1185 adults aged 21-47 years assessed in 1990). The predictive value of SPTs and RASTs on new-onset asthma and nasal allergy was assessed by Cox regression and by calculating the positive or negative predictive value.

During the 20 years between baseline and follow-up, 3.1 and 4.4 per 1000 person-years of new-onset asthma and nasal allergy cases were recorded, respectively. The hazard ratios for SPTs of any specific and of all aeroallergens combined were slightly higher than those of RASTs for asthma and nasal allergy. The negative predictive values of both the SPT and RAST were very high and similar (0.94-0.96), whereas the postive predictive values were low $(0.09-0.20)$.

Positive SPT results showed a better association with new onset asthma and nasal allergy than positive RAST either to any specific aeroallergens or to all combined.

@ERSpublications

Positive SPT shows better association with new-onset asthma and nasal allergy than positive radioallergosorbent tests http://ow.ly/qacPS

This article has supplementary material available from www.erj.ersjournals.com

Received: Jan 222013 | Accepted after revision: Aug 072013 | First published online: Sept 132013

Support statement: The work was supported by the Competence Network Asthma/COPD funded by the Federal Ministry of Education and Research (FKZ 01GI0881-0888) and the German Research Foundation (HE 3294/10-1, NO 262/7-1, MA 711/6-1).

Conflict of interest: Disclosures can be found alongside the online version of this article at www.erj.ersjournals.com

Copyright @ERS 2014 


\section{Introduction}

Asthma and nasal allergy are common chronic disorders affecting $2.0-8.4 \%$ and $12.1-34.4 \%$ of Europeans, respectively [1]. The prevalence of both conditions was increasing but appears more recently to have plateaued. Worldwide data indicate heterogeneity in asthma prevalence trends, whereas the prevalence of nasal allergy continues to increase in most countries [2]. Therefore, allergic diseases pose a serious public health problem with a substantial impact.

A few studies have prospectively assessed the incidence of asthma and/or nasal allergy in adulthood using a 10-year follow-up [3-6]. Most of these papers have utilised data from the European Community Respiratory Health Survey (ECRHS). No study has yet reported on the onset of asthma and nasal allergies in adulthood using a follow-up of $\geqslant 15$ years. While the prevalence of asthma and nasal allergy has been reported previously in adult German populations [7, 8], there are no studies providing incidence rates for Germany utilising a long-term follow-up of 20 years. Previous studies $[3,4,9,10]$ have identified sex, smoking, atopy, body mass index, bronchial hyperresponsiveness (BHR), exposure to mould and early acute respiratory infection as risk factors for asthma and nasal allergy. However, no study has considered the potential predictive value of skin prick tests (SPTs) or radioallergosorbent tests (RASTs) as determinants for new asthma and nasal allergy onset in adults. The longitudinal predictive power of these factors should be examined.

Our study presents prevalence and incidence data for asthma and nasal allergy in a German prospective cohort followed for 20 years. We also examined potential long-term predictors of new-onset asthma and nasal allergy, with a special emphasis on the role of SPT and RAST positivity.

\section{Material and methods \\ Study population and design}

The ECRHS is a population-based multi-centre cohort study of respiratory health among adults. Study design and methods have been described previously [11], and the full protocol is available at www.ecrhs.org. Two German centres, located in Hamburg and Erfurt, were included in this study. Briefly, ECRHS I (baseline study) was performed between 1990 and 1992 on random samples of the general population. In stage 1, each participant was sent a screening questionnaire. From the responders, a random sample was invited to take part in a clinical examination (stage 2). ECRHS II (2000-2002) and ECRHS III (2010-2012) are follow-up studies of the population included in ECRHS I stage 2. Data presented in this study were collected between 1990 and 2012 in the German centres in Hamburg and Erfurt, as part of the framework of ECRHS. In ECRHS I, 1282 and 1251 individuals participated in the Erfurt and Hamburg cohorts, respectively (fig. 1). In Erfurt, 16 individuals did not consent to address storage and thus only 1266 individuals were contacted for ECRHS III. In Hamburg, a randomly drawn subsample of 900 individuals was invited to participate in ECRHS III. Of the contacted individuals in Erfurt and Hamburg, 667 and 518, respectively, were between 21-47 years at baseline in ECRHS I. Therefore, our study population consists of 1185 males and females aged 21-47 years who participated in the medical examination at baseline. Of those, 924 subjects ( 540 from Erfurt and 384 from Hamburg) participated in the ECRHS III (responders) and 261 persons did not (nonresponders). To identify a population at risk of new-onset asthma and or nasal allergy, those who reported a history of asthma or nasal allergy in ECRHS I were excluded (asthma: 17 out of 23 in Erfurt or Hamburg and nasal allergy: 68 out of 89 in Erfurt or Hamburg). Within the remaining population, new-onset asthma and nasal allergy were defined as a positive response to the questions: "Have you ever had asthma?" and "Do you have any nasal allergies, including hay fever?", respectively. The age of new-onset asthma and nasal allergy was lower than the age at baseline (ECRHS I) for 29 and 26 participants, respectively. When this difference was not $>2$ years, the age of new-onset of disease was set equal to the age at baseline. For individuals for whom this difference was $>2$ years, the midpoint of the age of reported new onset of disease and age at ECRHS III was set as the age of new onset. In case the midpoint was still lower than the age at baseline or that no age of new onset of disease was reported, the subject was excluded. The final study population consisted of 874 subjects (517 from Erfurt and 357 from Hamburg) with no indication for asthma at baseline and 754 subjects (465 from Erfurt and 289 from Hamburg) with no indication of nasal allergies at baseline (fig. 1).

The study protocol was approved (ethical approval for baseline in 1989 (Medical Association of SchleswigHolstein in Bad Segeberg, Hamburg) and in 1991 (Erfurt) and for follow-up in 2009 (regarding both substudies)) by the Local Ethics Committee of the Bayerische Landesärztekammer and of the Medical Association of Schleswig-Holstein in Bad Segeberg. Written informed consent was obtained from all participants.

\section{Assessment of allergic sensitisation in ECRHS I}

SPTs were performed using Phazets (Pharmacia Diagnostics AB, Uppsala, Sweden), which are individual antigen-coated lancets, and two control lancets (histamine and uncoated). The lancets were applied to the 
a)

Erfurt

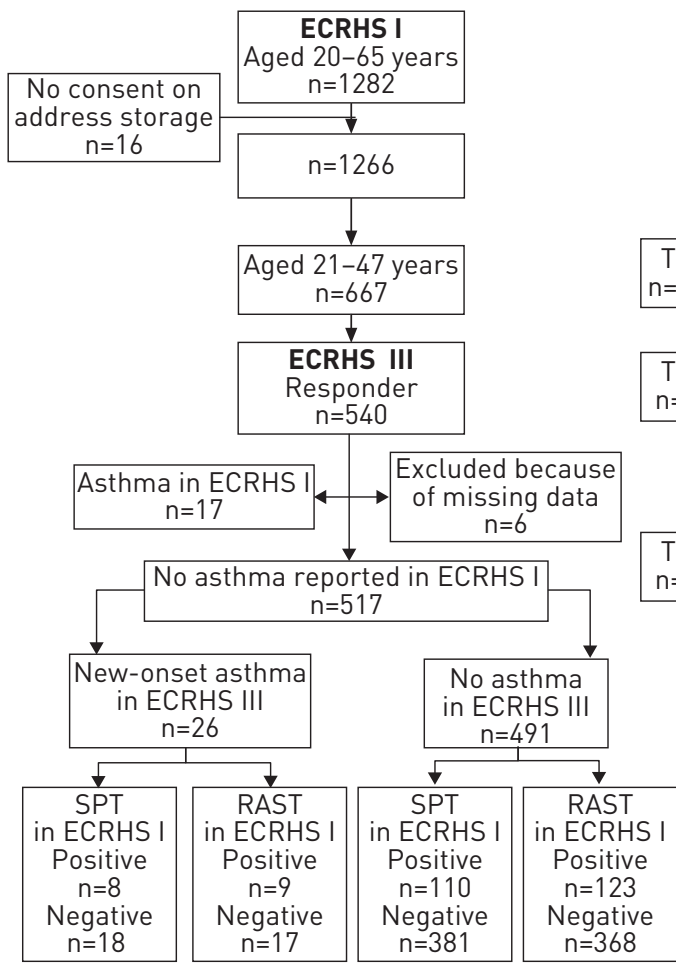

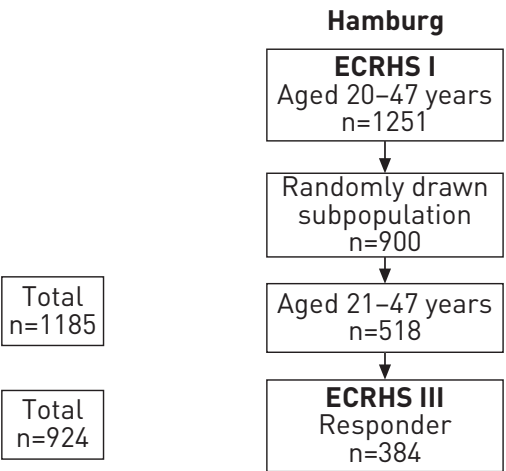

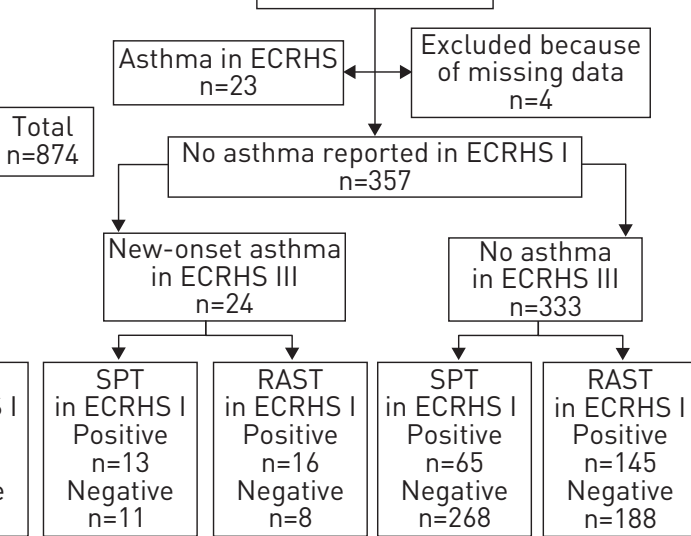

b)

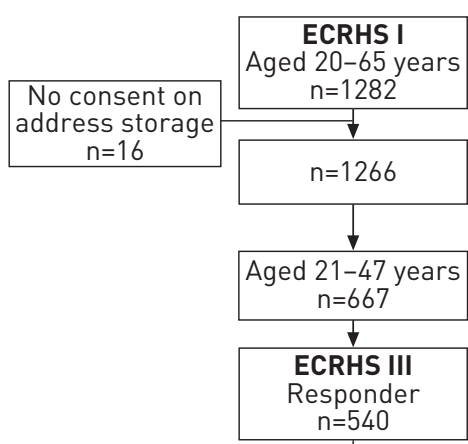

Erfurt

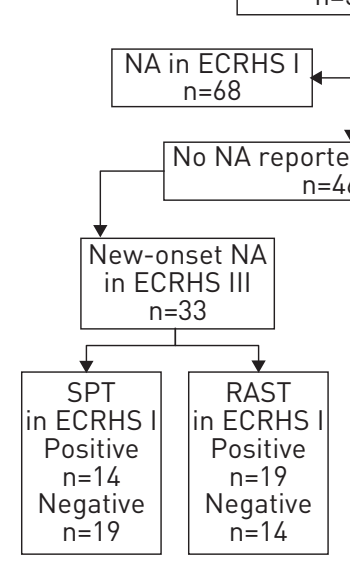

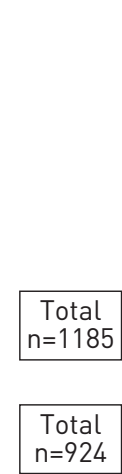

Hamburg

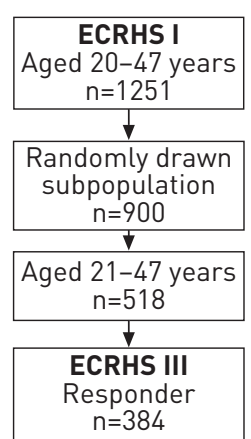

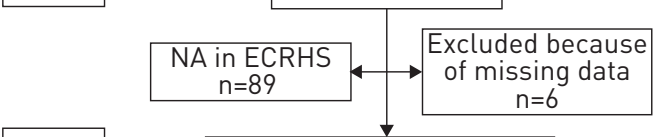
$\mathrm{n}=7$
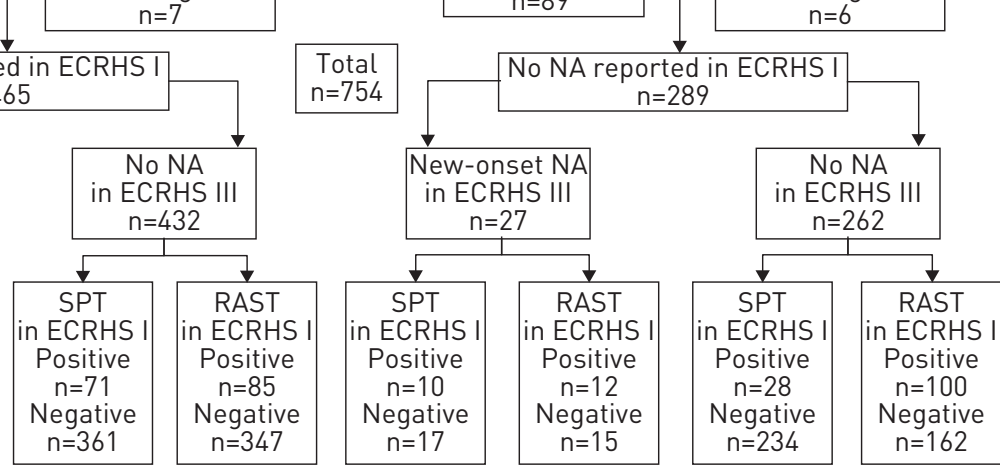

FIGURE 1 Flow chart for statistical analysis plan a) for asthma and b) for nasal allergy (NA). ECRHS: European Community Respiratory Health Survey; SPT: skin prick test; RAST: radioallergosorbent test. 
volar surface of the forearm. After $15 \mathrm{~min}$, the outline of the wheal was drawn on a strip of adhesive transparent tape, which was subsequently transferred to the data sheet. Wheal diameters were read at the widest point and at an angle of $90^{\circ}$ to the diameter at the midpoint. A skin test for a specific allergen was considered positive if a mean wheal diameter to the allergen was $\leqslant 3 \mathrm{~mm}$, and the wheal diameter to histamine was $\geqslant 3 \mathrm{~mm}$ and to uncoated Phazets $<3 \mathrm{~mm}$. Cat, Dermatophagoides pteronyssinus, Cladosporium herbarum and birch allergens were selected for this analysis.

Specific IgE against these same four allergens was also measured in serum samples in a central laboratory by RAST (CAP-RAST; Pharmacia Diagnostics). Values $>0.35 \mathrm{kU} \cdot \mathrm{L}^{-1}$ for an allergen were considered positive. Specific IgE against Timothy grass was not assessed as we wanted a similar allergen spectrum for both the SPTs and RASTs. Of the individuals with single RAST positivity for Timothy grass, $8.5 \%$ (five out of 59) and $27.8 \%$ (10 out of 36 ) had new-onset asthma and nasal allergy, respectively.

\section{Lung function measurements and BHR in ECRHS I}

In ECRHS I, forced expiratory volume in $1 \mathrm{~s}$ (FEV1) was determined using pneumatograph-based electronic spirometers (Compact Pneumo Laboratory, and PSC-PC, Jaeger, Würzburg, Germany). BHR was obtained using the metacholine challenge (Mefar MB3 dosimeter; Mefar srl, Bovezzo, Italy) and was defined as a fall of at least $20 \%$ in FEV1 associated with a cumulative dose of $\leqslant 2 \mathrm{mg}$ methacholine.

\section{Analysis of data}

Characteristics of responders and nonresponders at baseline were compared using Chi-squared tests. Asthma and nasal allergy incidence rates were calculated as the number of new cases of asthma and nasal allergy, respectively, divided by the person-years at risk during the observation period.

Relative risks with 95\% confidence intervals are given for potential determinants of new-onset asthma and nasal allergy. The following potential determinants were considered: sex, centre, asthma (for new-onset nasal allergy), nasal allergy (for new-onset asthma), eczema, BHR, family history (considered positive in the case of an affirmative answer to any of the following questions: "Did your mother ever have asthma?", "Did your mother ever have eczema, skin or nasal allergy or hay fever?", "Did your father ever have asthma?" and "Did your father ever have eczema, skin or nasal allergy or hay fever?"), pet ownership (considered positive in the case of an affirmative answer to any of the following questions: "Do you keep a cat?", "Do you keep a dog?" and "Do you keep any birds?"), mould exposure ("Has there ever been any mould or mildew on any surface, other than food, inside the home?"), job exposure ("Have you ever worked in a job which exposed you to vapours, gas, dust or fumes?"), current smoking, contact with older children in early life (considered positive in the case of reported older siblings or an affirmative answer to the question "Did you go to school, playschool or nursery with older children before the age of 5 years?"), any positive SPT (to cat, D. pteronyssinus, C. herbarum and birch), and any positive RAST (cat, D. pteronyssinus, C. herbarum and birch). Atopic status was determined using SPT and RAST. The social status of individuals was assessed using the reported age at which full time education was completed and was categorised into three groups: age $\leqslant 16$ years, $17-23$ years and $>23$ years. The population-attributable fraction was calculated as the risk difference divided by the risk in exposed (atopic) individuals multiplied by the proportion of all cases in the exposed (atopic) population.

Cox regression analyses were performed with person-years under observation as the dependent variable and asthma or nasal allergy as the event. Hazard ratios with $95 \%$ confidence intervals are given for predictive variables in crude models and in models adjusted for sex, centre, social status, family history and smoking.

Measures of diagnostic accuracy of SPTs and RASTs (positive predictive value (PPV), negative predictive value (NPV), sensitivity and specificity) were calculated for individuals who developed asthma or nasal allergies between 1990 and 2012. Positive likelihood ratio (LR+) was determined by the calculation of sensitivity/(1-specificity) and negative likelihood ratio (LR-) by the calculation of (1-sensitivity)/specificity. The significance level was set to 5\%. Kaplan-Meier curves were obtained with SigmaPlot 12.0 (Systat Software, Inc., San Jose, CA, USA). Venn diagrams were plotted using the Venn Diagram Plotter Program originally written by Kyle Littlefield for the Department of Energy (PNNL, Richland, WA, USA). All statistical analyses were performed with SAS 9.2 (SAS Institute, Cary, NC, USA).

\section{Results}

Descriptive data

Of the 1185 adults asked to participate in ECRHS III, 924 responded (response rate 78.0\%). In general, responders and nonresponders did not differ in their basic characteristics, as assessed in 1990-1992 during ECRHS I (table 1). However, responders were more likely to be female, from Erfurt and be of higher social status than nonresponders. In contrast, responders were less likely to own pets and currently smoke. 
TABLE 1 Characteristics of the European Community Respiratory Health Survey (ECRHS) study population, aged 21-47 years, at baseline (1990-1992), stratified by participation in ECRHS III

\begin{tabular}{|c|c|c|c|}
\hline & Follow-up participants & Follow-up nonparticipants & p-value \\
\hline Subjects $\mathbf{N}$ & 924 & 261 & \\
\hline Sex female & 464/924 (50.2) & $113 / 261(43.3)$ & 0.05 \\
\hline Mean age years & 34.6 & 33.9 & $0.18^{\# \#}$ \\
\hline Erfurt Centre & $540 / 924(58.4)$ & $127 / 261(48.7)$ & 0.01 \\
\hline Asthma at baseline & $40 / 924(4.3)$ & $6 / 261(2.3)$ & 0.13 \\
\hline Nasal allergy at baseline & $157 / 924(17.0)$ & $43 / 261(16.5)$ & 0.84 \\
\hline Eczema at baseline & $329 / 924(35.6)$ & $93261(35.6)$ & 0.99 \\
\hline BHR at baseline & $153 / 778(19.7)$ & 44/185 (23.8) & 0.21 \\
\hline Family history ${ }^{\#}$ & $245 / 914(26.8)$ & $68 / 255(26.7)$ & 0.96 \\
\hline Social status & & & 0.02 \\
\hline$\leqslant 16$ years & $18 / 856(2.1)$ & 12/233 (5.1) & \\
\hline $17-23$ years & $650 / 856(75.9)$ & $179 / 233(76.8)$ & \\
\hline 23 years & $188 / 856(22.0)$ & 42/233 (18.0) & \\
\hline Pet ownership at baseline & $276 / 924(29.9)$ & $99 / 259(38.2)$ & 0.01 \\
\hline Mould exposure ever & $208 / 916(22.7)$ & $59 / 259(22.8)$ & 0.98 \\
\hline Job exposure ${ }^{+}$ever & $396 / 923(42.9)$ & $120 / 261(46.0)$ & 0.38 \\
\hline Current smoking at baseline & $352 / 924(38.1)$ & $117 / 261(44.8)$ & 0.05 \\
\hline Any positive $\mathrm{SPT}^{\S}$ & $93 / 924(10.1)$ & $25 / 261(9.6)$ & 0.82 \\
\hline SPT cat & $27 / 924(2.9)$ & $10 / 261(3.8)$ & 0.46 \\
\hline SPT Dermatophagoides pteronyssinus & $46 / 924(5.0)$ & $16 / 261(6.1)$ & 0.46 \\
\hline SPT Cladosporium herbarum & $1 / 924(0.1)$ & $0 / 291(0.0)$ & 0.60 \\
\hline SPT birch & $56 / 924(6.1)$ & $14 / 261(5.4)$ & 0.67 \\
\hline Any positive RAST $^{f}$ & $318 / 924(34.4)$ & $107 / 261(41.0)$ & 0.05 \\
\hline RAST cat & $63 / 840(7.5)$ & $11 / 212(5.2)$ & 0.24 \\
\hline RAST D. pteronyssinus & $127 / 823(15.4)$ & $30 / 205(14.6)$ & 0.78 \\
\hline RAST C. herbarum & $50 / 838(6.0)$ & $7 / 213$ (3.3) & 0.12 \\
\hline RAST birch & $80 / 826(9.7)$ & $18 / 208(8.7)$ & 0.65 \\
\hline Contact with older children in early life & $709 / 924(76.7)$ & $203 / 261(77.8)$ & 0.72 \\
\hline
\end{tabular}

\section{Outcome data}

In total, 50 cases of new-onset asthma and 60 cases of new-onset nasal allergy occurred during the 20-year observation period (1990/1992-2010/2012), which corresponds to 16072 and 13668 person-years at risk, respectively.

\section{Main results}

The incidence rates for asthma and nasal allergy are presented in table 2. The asthma crude incidence rate was 3.11 per 1000 person-years and that for nasal allergy was 4.39 per 1000 person-years. In Hamburg, the incidence rate was higher than in Erfurt for both outcomes. Rates were also higher for females than males. At the end of the follow-up period, $44 \%$ of the new asthma cases and $10.3 \%$ of subjects without asthma stated that they had suffered from wheeze in the last 12 months. Additionally, 28 (56\%) of the incident asthma cases reported current treatment with asthma medication and thus might have been free of symptoms. Of those not undergoing current treatment with asthma medication, $33.3 \%$ of the subjects with new-onset asthma and 9.7\% without asthma reported wheeze symptoms in the last 12 months.

The relative risk and 95\% confidence intervals for the relationship between each risk factor with new-onset asthma and nasal allergy are given in table 3 . These findings differ slightly when the analyses are stratified by centre (online supplementary tables S1a and S2b).

The hazard ratios obtained by Cox regression, which were adjusted for sex, centre, social status, family history and smoking, were higher for each SPT compared with the corresponding RAST for both new-onset asthma and nasal allergy (table 4). A significantly increased hazard for new-onset asthma showed a positive RAST for cat, as well as positive SPTs for cat, D. pteronyssinus and birch. Positive SPTs for cat and birch, as well as a positive RAST for birch were significantly associated with an increased hazard for new-onset nasal 
TABLE 2 New-onset asthma in subjects without asthma and new-onset nasal allergy in subjects without nasal allergy during the 20-year follow-up period

New onset asthma subjects without asthma at ECRHS I incidence rate per 1000 person-years
New onset nasal allergy subjects without nasal allergy at ECRHS I incidence rate per 1000 person-years

\begin{tabular}{|c|c|c|}
\hline Overall & 3.11 & 4.39 \\
\hline Hamburg & 3.65 & 5.14 \\
\hline Erfurt & 2.74 & 3.92 \\
\hline Female & 4.06 & 5.26 \\
\hline
\end{tabular}

ECRHS: European Community Respiratory Health Survey.

TABLE 3 Unadjusted predictors for the new-onset of asthma and nasal allergy in German adults during a 20-year follow-up

New-onset asthma subjects without asthma at ECRHS I

New-onset nasal allergy subjects without nasal allergy at ECRHS I

\begin{tabular}{|c|c|c|}
\hline Subjects $\mathrm{n}$ & 874 & 754 \\
\hline \multicolumn{3}{|l|}{ Sex } \\
\hline Female & $1.79(1.02-3.15)$ & $1.44(0.88-2.35)$ \\
\hline Male & 1 & 1 \\
\hline Erfurt & $0.75(0.44-1.28)$ & $0.76(0.47-1.24)$ \\
\hline Hamburg & 1 & 1 \\
\hline Asthma at baseline & & $1.75(0.59-5.16)$ \\
\hline No nasal allergy at baseline & 1 & \\
\hline Eczema at baseline & $1.62(0.95-2.78)$ & $1.76(1.09-2.86)$ \\
\hline No eczema at baseline & 1 & 1 \\
\hline BHR at baseline & $4.14(2.29-7.48)$ & $1.69(0.96-2.99)$ \\
\hline No BHR at baseline & 1 & 1 \\
\hline \multicolumn{3}{|l|}{ Family history\# } \\
\hline $17-23$ years & $0.33(0.11-0.98)$ & $1.23(0.18-8.37)$ \\
\hline$>23$ years & $0.19(0.05-0.69)$ & $1.20(0.17-8.60)$ \\
\hline Pet ownership at baseline & $0.91(0.50-1.66)$ & $0.85(0.49-1.47)$ \\
\hline No pet ownership at baseline & 1 & 1 \\
\hline Mould exposure ever & $0.77(0.38-1.57)$ & $1.49(0.88-2.52)$ \\
\hline No mould exposure ever & 1 & 1 \\
\hline Job exposure ${ }^{+}$ever & $1.05(0.61-1.80)$ & $0.89(0.54-1.47)$ \\
\hline No job exposure ever & 1 & 1 \\
\hline Current smoking at baseline & $0.71(0.39-1.27)$ & $0.63(0.37-1.07)$ \\
\hline No current smoking at baseline & 1 & 1 \\
\hline Any positive SPT ${ }^{\S}$ & $2.51(1.46-4.29)$ & $3.42(2.12-5.52)$ \\
\hline No positive SPT & 1 & 1 \\
\hline Any positive RAST $^{f}$ & $1.98(1.16-3.39)$ & $2.66(1.65-4.31)$ \\
\hline
\end{tabular}


TABLE 4 Adjusted associations between skin prick tests (SPTs) and radioallergosorbent test (RAST) and the new onset of asthma and nasal allergy during a 20-year follow-up

New-onset asthma subjects without asthma at ECRHS I
New-onset nasal allergy subjects without nasal allergy at ECRHS I

\begin{tabular}{|c|c|c|}
\hline Subjects $\mathrm{n}$ & 874 & 754 \\
\hline SPT cat & $5.46(2.59-11.49)$ & $6.60(2.94-14.84)$ \\
\hline RAST cat & $2.52(1.04-6.08)$ & $2.01(0.79-5.10)$ \\
\hline SPT Dermatophagoides pteronyssinus & $2.23(1.02-4.88)$ & $1.82(0.85-3.88)$ \\
\hline SPT Cladosporium herbarum & $3.63(0.86-15.37)$ & $1.34(0.18-9.88)$ \\
\hline RAST C. herbarum & $0.93(0.22-3.90)$ & $1.18(0.37-3.79)$ \\
\hline SPT birch & $2.19(1.08-4.44)$ & $7.24(3.91-13.39)$ \\
\hline Any positive RAST & $1.90(1.01-3.58)$ & $3.43(1.90-6.19)$ \\
\hline
\end{tabular}

Data are presented as hazard ratio $(95 \% \mathrm{Cl})$ adjusted for sex, centre, social status, family history and smoking, unless otherwise stated. ECRHS: European Community Respiratory Health Survey. ${ }^{\#}$ : at least one positive SPT; ${ }^{\natural}$ : at least one positive RAST.

allergy. The highest hazard ratio was observed between a positive SPT for cat and new-onset asthma as well as between a positive SPT for birch and new-onset nasal allergy. Any positive SPT and any positive RAST significantly increased the hazard for new-onset asthma and nasal allergy. Thus, for both outcomes, the hazard ratio was stronger for any positive SPT than for any positive RAST. When the analysis was stratified by centre, this trend was similar in Hamburg but reversed in Erfurt. However, the numbers in both of these stratified analyses are small and there is a substantial overlap in the confidence intervals (online supplementary tables S1a-S4b).

As seen in figure 2, there is partial overlap between individuals with new-onset asthma and nasal allergy who were positive to any SPT and any RAST. Among those with either new-onset asthma or nasal allergy, more individuals tested positive for any RAST than for any SPT. In total, 31 (62\%) out of 50 individuals with new-onset asthma and $38(63 \%)$ out of 60 individuals with new-onset nasal allergy tested positive for at least one SPT and/or at least one RAST at baseline and, therefore, were atopic. The population attributable fraction for asthma and nasal allergy was $36 \%$ and $44 \%$, respectively.

Of the 874 nonasthmatic individuals at baseline, 196 were positive to at least one SPT, 21 of whom developed asthma during the 20-year observation period. Similarly, 293 of the 874 nonasthmatics were positive to at least one RAST, 25 of whom developed asthma. With regard to nasal allergy, 754 individuals were without nasal allergies at baseline, 123 of whom were positive to at least one SPT and 24 of whom subsequently developed nasal allergies during the follow-up period. Finally, 216 (of the 754 individuals without nasal allergies) were positive to at least one RAST, 31 of whom developed nasal allergies during follow-up. Sensitivity was higher for RAST than for SPT for both asthma (50.0\%, 95\% CI 36.1-63.9\% versus $42.0 \%, 95 \%$ CI $28.3-55.7 \%)$ and nasal allergy $(51.6 \%, 95 \%$ CI $39.0-64.3 \%$ versus $40.0 \%$, 95\% CI 27.6-52.4\%). Although the confidence intervals are overlapping specificity was higher for SPT than for RAST for asthma $(78.8,95 \%$ CI 76.0-81.6 versus $67.5,95 \%$ CI $64.3-70.7)$ and for nasal allergy $(85.7,95 \%$ CI 83.1-88.3 versus $73.3,95 \%$ CI 70.1-76.6). As seen in table 5, the PPVs for SPT and RAST for asthma were very low and similar and the confidence intervals overlapped $(0.11,95 \%$ CI $0.07-0.16$ versus $0.9,95 \%$ CI 0.06-0.12). For nasal allergy, the PPVs for SPT and RAST were low and differed more substantially, although again the confidence intervals overlapped $(0.2,95 \%$ CI $0.13-0.28$ versus $0.14,95 \%$ CI $0.10-0.20)$. The NPVs for SPT and RAST were high and similar for asthma and nearly identical for nasal allergy.

LR+ and LR- for SPTs and RASTs were rather weak, as LR+ was (with the exception of SPTs in nasal allergy, where the LR+ was 2.8) below 2 and LR- was between 0.5 and 1.0 for both tests (table 5).

The relationship between time to onset of asthma/nasal allergy and new-onset asthma/nasal allergy for subjects with any positive SPT and subjects with any positive RAST at baseline is shown in figure 3. 2 years after the baseline survey (ECRHS I), the probability of new-onset asthma was greater for individuals with at least one positive SPT than for those with at least one positive RAST. For all time-points, the probability of new-onset nasal allergy was higher for individuals with at least one positive SPT than for individuals with at least one positive RAST. 
a)

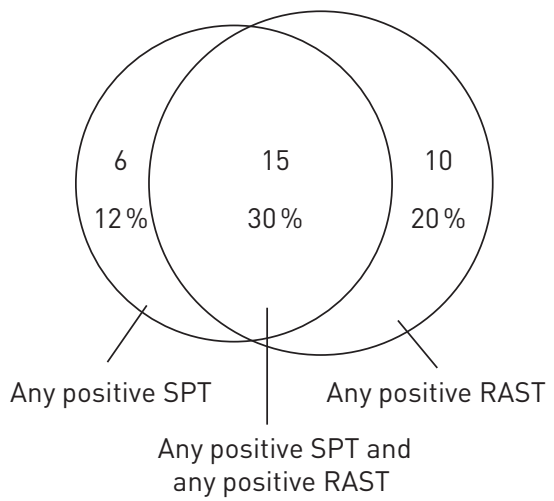

b)

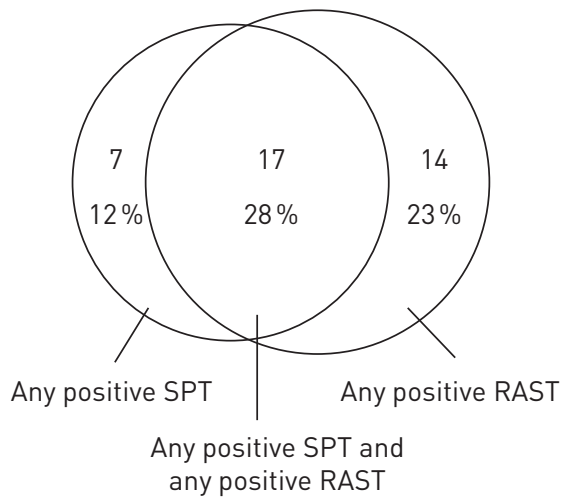

FIGURE 2 Venn diagram depicting the overlap between any positive skin prick test (SPT) and any positive radioallergosorbent test (RAST) at baseline (1990-1992) in patients with a) a new onset of asthma $(\mathrm{N}=50)$ or b) nasal allergy $(\mathrm{N}=60)$ in European Community Respiratory Health Survey III. Any positive SPT: at least one positive SPT; Any positive RAST: at least one positive RAST.

\section{Discussion}

\section{Key results}

The present study provides information about the incidence, risk factors and prediction by SPT and RAST of new-onset asthma and nasal allergy in a population of German adults followed for 20 years.

The incidence rates for asthma and nasal allergy were higher in Hamburg than in Erfurt. Females had a higher incidence rate than males for both conditions. The incidence of asthma was related to sex, BHR, nasal allergy, social status, any positive SPT and any positive RAST. The incidence of nasal allergy was associated with eczema, any positive SPT and any positive RAST. SPT was a slightly better predictor for the new onset of asthma and nasal allergy than RAST. An analysis of allergen-specific SPT and RAST identified a positive reaction to the cat allergen as the strongest predictor for new-onset asthma and a positive reaction to the birch allergen as the strongest predictor for new-onset nasal allergy.

\section{Limitations}

The main limitation of long-term prospective cohort studies is a loss to follow-up. However, the response rate of our study in ECRHS III can be regarded as high ( $78 \%$ when only eligible subjects are considered). Responders did not differ from nonresponders in many potential risk factors, such as age, nasal allergy, eczema, SPT or RAST. Nevertheless, our sample cannot be considered as completely unbiased. The long observation period of our study, with time intervals of $\sim 10$ years between follow-ups, might lead to recall bias, especially in terms of age of new-onset of disease. We used self-reported data from questionnaires to assign asthma and nasal allergy status, which may lead to misclassification, especially with regard to asthma, given the potential confounding influence of chronic obstructive pulmonary disease. We think asthma medication, as an outcome, is likely to be less subject to recall bias than self-reported physician diagnosis of asthma or nasal allergy. Including more self-reported respiratory symptoms as outcomes would have similar limitations. More "objective" data, such as lung function measurements and BHR testing or a clinical case ascertainment, would diminish the misclassification bias of this study. Unfortunately, this information was

TABLE 5 Measures of diagnostic accuracy regarding skin prick test (SPT) and radioallergosorbent test (RAST) as predictors of new-onset asthma and nasal allergy

Asthma ${ }^{\#}$

Asthma"

\begin{tabular}{lccccc} 
& SPT & RAST & SPT & RAST \\
\cline { 2 - 5 } & 1.98 & 1.54 & 0.80 & 1.94 \\
LR+ & 0.73 & 0.74 & 0.69 & 0.66 \\
LR- & $0.11(0.07-0.16)$ & $0.09(0.06-0.12)$ & & $0.20(0.13-0.28)$ & $0.14(0.10-0.20)$ \\
PPV (95\% Cl) & $0.96(0.94-0.97)$ & $0.96(0.94-0.97)$ & & $0.94(0.92-0.96)$ & $0.95(0.92-0.96)$ \\
NPV (95\% Cl) & &
\end{tabular}

LR+: positive likelihood ratio; LR-: negative likelihood ratio; PPV: positive predictive value; NPV: negative predictive value. ${ }^{\#}: \mathrm{n}=874 ;{ }^{\top}: \mathrm{n}=754$. 

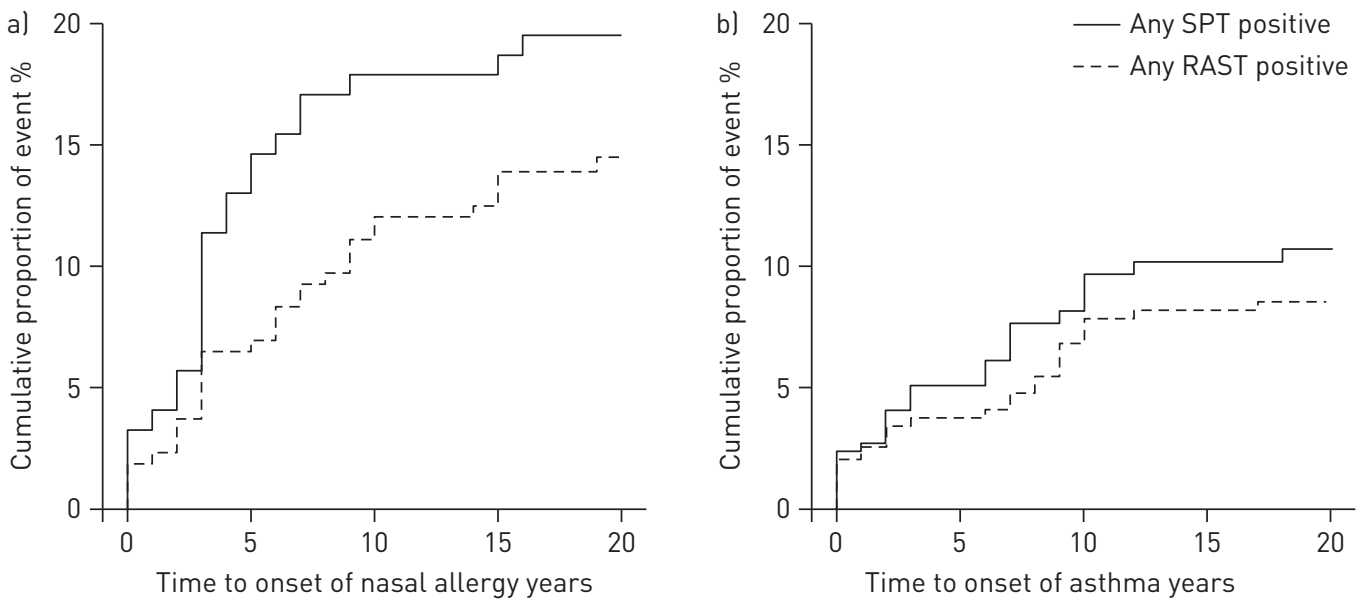

FIGURE 3 Kaplan-Meier curves for new-onset of asthma and nasal allergy shown as the cumulative proportion of newonset asthma/nasal allergy at certain times under control against the time to onset of a) nasal allergy or b) asthma. Participants were from Erfurt and Hamburg and were aged 21-47 years at baseline. SPT: skin prick test; RAST: radioallergosorbent test; any SPT positive: at least one positive skin prick test; any RAST positive: at least one positive radioallergosorbent test.

not available. Also, it is possible that some of the new-onset asthma cases are attributable to a change in diagnostic labelling.

14 and 46 individuals, respectively, reported that they ever had asthma and nasal allergy in ECRHS I but did not report these symptoms in ECRHS III. These inconsistencies could be due to a true remission in individuals who have had asthma or nasal allergy years ago, but that the disease did not persist. Considering the long observation period of this study, it is plausible that some individuals with new-onset asthma or nasal allergy who had negative SPT and RAST at baseline became sensitised during the observation period. Furthermore, a lower cut-off point for the tests might lead to a higher sensitivity.

The large sample size, international standardised methods of data collection following ECRHS protocols and he population-based setting of this study are unique and important strengths. Moreover, the design of a prospective cohort with a long (20-year) follow-up time, which includes a question on new onset, allows incidence rates to be calculated. The detailed information on health behaviours and risk factors, gained through questionnaires and medical examinations, permits analyses to be adjusted for a large number of potential confounders.

\section{Interpretation}

Incidence rates of asthma (crude 3.1, males 2.2 and females 4.1) were within the range of those observed in previous cohort studies (crude 2.2-4.5, males 1.0-4.5 and females 1.3-4.9) [12-14]. As reported by most other studies, a higher incidence rate was found for females than for males [9, 10]. One study reported that only the risk of developing nonallergic asthma differed by sex [15].

In the current study, incidence rates of nasal allergy (crude 4.4, males 3.6 and females 5.3) are below the lifelong incidence reported by MATHESON et al. [4] in all 48 centres of the ECRHS study (7.0 males and 7.9 females). This difference might be due to the fact that our study does not report lifelong incidence but rather the incidence of nasal allergy between 1990 and 1992, and 2010 and 2012 only. This shorter timeframe probably leads to less recall and misclassification bias.

Although risk factors for asthma and nasal allergy have been assessed previously, this is the first cohort study to show the effect of potential determinants on new-onset of disease over a 20-year follow-up.

The association between low social status and new-onset asthma found in the present study confirms the results of previous publications that have reported similar associations with the prevalence of asthma [16], with the exception of the study by MONTNEMERY et al. [17]. The association between socioeconomic status and asthma in children is complex [18] and is likely to depend on asthma severity, poverty, many lifestyle factors and potentially also country of residence. While (probably severe) asthma prevalence is higher in children from less privileged families in inner cities, we previously found that asthma is more prevalent in the higher social classes in a large cohort of German children, which is consistent with other German studies [19]. 
The impact of atopy on asthma remains unclear. 20 years ago, BuRrows et al. [20] stated that asthma almost always has an allergic basis in children and adults. However, ANTO et al. [3] and ZACHARASIEWICZ et al. [21] found the attributable fraction of atopy on the development of asthma to be in the range of $12 \%$ and $30 \%$ in adults. The attributable fraction we report (36\%) is higher.

Among subjects with new onset of nasal allergy, 63\% were atopic (at least one positive SPT or RAST) at baseline. This finding is consistent with data published by ZACHARASIEWICZ et al. [21], who reported that $61 \%$ of rhinitis cases were atopic. However, their attributable fraction $(53 \%)$ is higher than that of the current study $(44 \%)$. The nonatopic cases may represent rhinitis without an allergic base or may represent cases who were not atopic at baseline but who developed atopy during the observation period. Furthermore, including Timothy grass in the RAST would probably have lead to higher attributable fractions for newonset asthma and nasal allergy.

SPT and RAST are both significant predictors for new-onset asthma and nasal allergy. The calculation of PPV and NPV demonstrated that positivity to at least one SPT is a better predictor for both conditions than positivity to at least one RAST. However, the overlapping confidence interval of these point estimates indicates that this potential superiority is likely to be minor. The negative prediction of at least one positive SPT and at least one positive RAST were (nearly) equal and performed better than the positive predictions. The sensitivity of RAST for new onset was stronger than of SPT, while specificity showed better results for SPT. Similar results were reported for both conditions for adults by Tschopp et al. [22] using Phadiatop instead of RAST and for children by SCHÄFER et al. [23] with regard to hay fever.

The likelihood ratio indicates the value of a diagnostic test by comparing the likelihood that a certain test result would be expected in a patient with the definite disorder with the likelihood of the same result expected in a patient without the definite disorder. Here, a distinction is drawn between LR+, concerning positive test results, and LR-, concerning negative test results. The classification of the test value given by the likelihood ratio is determined as excellent $(\mathrm{LR}+>10$ and LR- $<0.10)$, good (LR+ 5-10 and LR- 0.1-0.2), moderate ( LR+ 2-5 and LR- 0.2-0.5) or weak (LR+ 1-2 and LR- 0.5-1.0) [24]. Therefore, the value of SPT and RAST in terms of prediction of new-onset asthma and nasal allergy is poor. When interpreting the test values of SPT and RAST for new-onset asthma, it must be acknowledged that only a proportion of all asthma cases are atopic. Furthermore, the much lower rate of SPT positivity than RAST positivity might have an effect on these estimates. Sensitisation to cat allergen was most strongly associated with new-onset asthma, followed by mite. Considering the allergens tested in this study, for new-onset nasal allergy, sensitisation to birch allergen was the strongest predictor, followed by cat. Positive SPT of every single allergen was more strongly related to new-onset of both asthma and nasal allergy than a positive RAST. This association might even increase with the use of a lower cut-off value for SPT [23, 25]. Previous studies have stated that the results of SPT and RAST are not interchangeable and that a positive SPT not accompanied by a positive RAST might be due to IgE antibodies that are not detectable by any RAST procedures [26, 27]. As previously shown, there are differences in the prevalence of atopic diseases between east and west Germany $[7,28]$. Therefore, as anticipated, the current results differ between Hamburg and Erfurt when the analyses are stratified by centre. This observation supports the hypothesis of site-specific influences on risk factors for the new onset of asthma and nasal allergy. Although the sample sizes are small, there seems to be a generally higher risk of specific allergic sensitisation for asthma onset in Hamburg compared with Erfurt. Similar differences were not found for nasal allergies. As suggested by the International Study of Asthma and Allergies in Childhood [29], there might be substantial variation in the dependency between asthma and atopy, which could explain the differences in the associations between SPT and RAST with new onset of asthma and nasal allergy in Erfurt and Hamburg.

\section{Conclusion}

We report that SPT and RAST are both unpromising tests for prediction of new onset of asthma and nasal allergy in a cohort of German adults followed for 20 years, although a positive SPT seems to present a slightly better predictor than any detectable specific IgE. However, the role of atopy on these diseases remains ambiguous, especially with regard to asthma. Further prospective longitudinal studies are needed to determine long-term predictors for new-onset asthma and nasal allergy.

\section{Acknowledgements}

We wish to thank all participants in the study. We are indebted to the ECRHS study group and to all coworkers who were responsible for the design and conduct of the study.

\section{References}

1 Variations in the prevalence of respiratory symptoms, self-reported asthma attacks, and use of asthma medication in the European Community Respiratory Health Survey (ECRHS). Eur Respir J 1996; 9: 687-695. 
Asher MI, Montefort S, Bjorksten B, et al. Worldwide time trends in the prevalence of symptoms of asthma, allergic rhinoconjunctivitis, and eczema in childhood: ISAAC phases one and three repeat multicountry cross-sectional surveys. Lancet 2006; 368: 733-743.

3 Anto JM, Sunyer J, Basagana X, et al. Risk factors of new-onset asthma in adults: a population-based international cohort study. Allergy 2010; 65: 1021-1030.

4 Matheson MC, Dharmage SC, Abramson MJ, et al. Early-life risk factors and incidence of rhinitis: results from the European Community Respiratory Health Study - an international population-based cohort study. J Allergy Clin Immunol 2011; 128: 816-823.

5 Thomsen SF, Ulrik CS, Kyvik KO, et al. The incidence of asthma in young adults. Chest 2005; $127: 1928-1934$.

6 Toren K, Gislason T, Omenaas E, et al. A prospective study of asthma incidence and its predictors: the RHINE study. Eur Respir J 2004; 24: 942-946.

7 Nowak D, Heinrich J, Jorres R, et al. Prevalence of respiratory symptoms, bronchial hyperresponsiveness and atopy among adults: west and east Germany. Eur Respir J 1996; 9: 2541-2552.

8 Filipiak B, Heinrich J, Nowak D, et al. The distribution in specific IgE and the prevalence of allergic symptoms in 25-64-years old inhabitants of an eastern and a western German city - results from Augsburg and Erfurt. Eur J Epidemiol 2001; 17: 77-84.

9 Chinn S, Downs SH, Anto JM, et al. Incidence of asthma and net change in symptoms in relation to changes in obesity. Eur Respir J 2006; 28: 763-771.

10 de Marco R, Pattaro C, Locatelli F, et al. Influence of early life exposures on incidence and remission of asthma throughout life. J Allergy Clin Immunol 2004; 113: 845-852.

11 Burney PG, Luczynska C, Chinn S, et al. The European Community Respiratory Health Survey. Eur Respir J 1994; 7: 954-960.

12 Winer RA, Qin X, Harrington T, et al. Asthma incidence among children and adults: findings from the Behavioral Risk Factor Surveillance System Asthma Call-Back Survey - United States, 2006-2008. J Asthma 2012 ; 49: 16-22.

13 Lundback B, Ronmark E, Jonsson E, et al. Incidence of physician-diagnosed asthma in adults - a real incidence or a result of increased awareness? Report from The Obstructive Lung Disease in Northern Sweden Studies. Respir Med 2001; 95: 685-692.

14 Eagan TM, Bakke PS, Eide GE, et al. Incidence of asthma and respiratory symptoms by sex, age and smoking in a community study. Eur Respir J 2002; 19: 599-605.

15 Leynaert B, Sunyer J, Garcia-Esteban R, et al. Gender differences in prevalence, diagnosis and incidence of allergic and non-allergic asthma: a population-based cohort. Thorax 2012; 67: 625-631.

16 Ellison-Loschmann L, Sunyer J, Plana E, et al. Socioeconomic status, asthma and chronic bronchitis in a large community-based study. Eur Respir J 2007; 29: 897-905.

17 Montnemery P, Bengtsson P, Elliot A, et al. Prevalence of obstructive lung diseases and respiratory symptoms in relation to living environment and socio-economic group. Respir Med 2001; 95: 744-752.

18 Cruz AA, Bateman ED, Bousquet J. The social determinants of asthma. Eur Respir J 2010; 35: $239-242$.

19 Heinrich J, Popescu MA, Wjst M, et al. Atopy in children and parental social class. Am J Public Health 1998; 88: 1319-1324.

20 Burrows B, Martinez FD, Halonen M, et al. Association of asthma with serum IgE levels and skin-test reactivity to allergens. N Engl J Med 1989; 320: 271-277.

21 Zacharasiewicz A, Douwes J, Pearce N. What proportion of rhinitis symptoms is attributable to atopy? J Clin Epidemiol 2003; 56: 385-390.

22 Tschopp JM, Sistek D, Schindler C, et al. Current allergic asthma and rhinitis: diagnostic efficiency of three commonly used atopic markers (IgE, skin prick tests, and Phadiatop). Results from 8329 randomized adults from the SAPALDIA Study. Swiss Study on Air Pollution and Lung Diseases in Adults. Allergy 1998; 53: 608-613.

23 Schäfer T, Hoelscher B, Adam H, et al. Hay fever and predictive value of prick test and specific IgE antibodies: a prospective study in children. Pediatr Allergy Immunol 2003; 14: 120-129.

Altman DG, ed. Practical Statistics for Medical Research. London, Chapman \& Hall, 2001.

25 Bousquet PJ, Chatzi L, Jarvis D, et al. Assessing skin prick tests reliability in ECRHS-I. Allergy 2008; 63: 341-346.

26 Witteman AM, Stapel SO, Perdok GJ, et al. The relationship between RAST and skin test results in patients with asthma or rhinitis: a quantitative study with purified major allergens. J Allergy Clin Immunol 1996; 97: $16-25$.

27 van der Zee JS, de Groot H, van Swieten P, et al. Discrepancies between the skin test and IgE antibody assays: study of histamine release, complement activation in vitro, and occurrence of allergen-specific IgG. J Allergy Clin Immunol 1988; 82: 270-281.

28 Heinrich J, Richter K, Magnussen H, et al. Is the prevalence of atopic diseases in east and west Germany already converging? Eur J Epidemiol 1998; 14: 239-245.

29 Mallol J, Crane J, von Mutius E, et al. The International Study of Asthma and Allergies in Childhood (ISAAC) Phase Three: a global synthesis. Allergol Immunopathol (Madr) 2013; 41: 73-85. 